\title{
Preparation and Characterization of Hypoxia Sensitive Cationic Liposomal Doxorubicin and Evaluation Its Anti-tumor Activity in Mice Bearing C26 Tumors
}

\section{Mohammad Mashreghi}

Mashhad University of Medical Sciences https://orcid.org/0000-0002-5197-6260

\section{Mahdi Faal Maleki}

Mashhad University of Medical Sciences

Anis Askarizadeh

Mashhad University of Medical Sciences

Helaleh Farshchi

Mashhad University of Medical Sciences

Leila Farhoudi

Mashhad University of Medical Sciences

Mahda Sadat Nasrollahzadeh

Mashhad University of Medical Sciences

Farzin Hadizadeh

Mashhad University of Medical Sciences

Mahmoud Reza Jaafari ( $\boldsymbol{\nabla}$ jafarimr@mums.ac.ir)

https://orcid.org/0000-0003-3908-6828

\section{Nano Express}

Keywords: Tumor microenvironment, hypoxia, azo-linker, cationic liposome, doxorubicin

Posted Date: August 30th, 2021

DOI: https://doi.org/10.21203/rs.3.rs-832393/v1

License: (c) (i) This work is licensed under a Creative Commons Attribution 4.0 International License. Read Full License 


\section{Abstract}

The goal of this study was to prepare cationic nanoliposomal doxorubicin in which PEG molecule attached to the liposome via a hypoxia-sensitive azo linker. The cost-effective hypoxia-sensitive molecule (HSM) was synthesized composing of $\mathrm{C}_{18} \mathrm{H}_{37}$ lipophilic tail, azo-linker, and $\mathrm{PEG}_{2000}$ hydrophilic molecule. The NMR and FTIR were employed to characterize the HSM. Then, this compound was post-inserted into the cationic liposome (Cat-lip), and PEG-Azo-Cat-lip was prepared and characterized using DLS. In vitro release and cytotoxicity studies were performed in normoxic and hypoxic conditions. In vivo biodistribution and anti-tumor activities of the formulations were studied on mice bearing C-26 colon carcinoma tumor model and compared with PEGylated liposomal doxorubicin (Caelyx ${ }^{\circledR}$ ). Besides, the histological test confirmed the formulation biosafety on healthy mice. The results of NMR and FTIR indicated the synthesis of HSM. The decrease in the zeta-potential of formulation from $+18.4 \mathrm{mV}$ for Catlip to $+6.1 \mathrm{mV}$ along with the increase in the size of the PEG-Azo-Cat-lip indicated the successful postinsertion of HSM. The release study showed that PEGylation results in the more stable PEG-Azo-Cat-lip compared to the Cat-lip. The increased cytotoxicity of the PEG-Azo-Cat-lip in the hypoxic condition also indicated the cleavage of the azo-linker in the hypoxic environment. In vivo biodistribution using animal imaging has shown higher tumor accumulation of the PEG-Azo-Cat-lip than Cat-lip during the $120 \mathrm{~h}$ of the study. The results of anti-tumor activities and biosafety of the formulations also showed the higher efficiency of the PEG-Azo-Cat-lip compared with the Cat-lip. The results of this study, indicated the antitumor efficacy of this hypoxia-sensitive which merits further investigation.

\section{Introduction}

Nanocarriers improve the bioavailability and efficiency of conventional chemotherapeutics along with decreasing theirs off-target and undesirable side-effects [1]. The phenomenon so-called enhanced permeation and retention (EPR) effect explaining how nanocarriers accumulate in the tumor site. In this way, leaky pathophysiological blood vessels and deficiency in lymphatic drainage result in the accumulation of the nanocarriers with an average size of $100 \mathrm{~nm}$ in the tumor microenvironment [2]. Despite the success of the nano-drug delivery systems (NDDSs), there are emerging researches that claim only small amounts of chemotherapeutics passively delivered to the desired site of action [3]. Cationic liposomes are among the NDDSs with the high ability to uptake by the cancer cell in vitro, due to their positively charged surface. In theory, cationic liposomes have a high tendency to the tumor microenvironment, especially tumor vasculature [4]. However, despite the in vitro success, the in vivo administration of the cationic liposome resulted in the rapid clearance by the reticuloendothelial system (RES) due to protein opsonization [5]. PEGylation and making stealth liposome resulted in the long blood circulation of the liposome but also in case of positively-charged liposome cover their cationic properties.

Presently, scientists are trying to use active targeting methods to address the mentioned drug delivery's issues $[6,7]$. Designing NDDSs with the ability to respond to the endogenous and/or exogenous stimuli is one of the most known forms of active targeting strategies [8]. Stimuli-responsive NDDSs are stable in the blood circulation, however, they become unstable in response to the stimuli. This phenomenon leads 
to reduce side effects due to avoiding unwanted drug release. The two main groups of stimuli are exogenous (temperature, light and radiofrequency) and endogenous ( $\mathrm{pH}$, enzyme and hypoxia), which NDDS release its payload in response to them [9]. Pathophysiological conditions of the tumor microenvironment give rise to the changes in factors, including concentration of the $\mathrm{H}^{+}$ions, enzymes that present in the extracellular matrix (ECM), changes in the reduction/oxidation (redox) status, and induction of the hypoxia $[10,11]$. By increasing our understanding of the changes that occur in the tumor microenvironment, we will be able to design, synthesize and insert chemical structures as hypoxiasensitive linkers into the NDDS which will disrupt the stability of the NDDS in desired sites of action.

The fast-growing ability of cancer cells results in the formation of low oxygen supply regions known as hypoxia areas. As one of the hallmarks of cancer, angiogenesis is responsible for providing fast-growing tumor cells with nutrients and oxygen [12]. Intensive researches have been done to discover the mechanisms involved in angiogenesis in tumor microenvironments [13]. One of the most critical signals in the formation of new blood vessels is vascular endothelial growth factor (VEGF) and its receptors (VEGFR), which in turn, are affected by the hypoxia-inducible factor (HIF) [8]. The hypoxic areas are the main initiators of the successive above-mentioned stages and the formation of new blood vessels [14]. In this regard, azo-based molecules have the ability to target hypoxic regions and have been used in azoderivative chemotherapeutics as theragnostic agents in cancer $[15,16]$. It was revealed that azobenzene derivatives have reduction potentials in the range of that of hypoxic environments, which leads to reduction and consequently cleaving of $\mathrm{N}=\mathrm{N}$ bonds [17].

The aim of this study was to design and synthesize cationic nanoliposomal doxorubicin (Dox), in which PEG molecule was attached to the liposome via a hypoxia-sensitive azo linker. In hypoxic tumor environment, the PEG molecule will be cleaved in response to the hypoxic condition and releasing PEG molecule resulted in the exposure of positively-charged cationic liposome to the tumor cells and enhancing anti-tumor activity. In blood PEG molecule plays a role as coverage, which makes cationic liposome stealth and avoid RES.

\section{Materials And Methods}

\section{Materials}

All intermediates and target hypoxia-sensitive linker were characterized by FT-IR, ${ }^{1} \mathrm{H}$ NMR spectra. ${ }^{1} \mathrm{H}$ NMR $\left(300 \mathrm{MHz}\right.$ ) was obtained by Bruker FT-300 MHz instrument (Karlsruhe, Germany), $\left(\mathrm{CDCl}_{3}, \mathrm{DMSO} \mathrm{d}_{6}\right)$. Perkin Elmer 1420 spectrometer (Massachusett, USA) was exploited to obtain infrared spectra applying $\mathrm{KBr}$ disks. 1-Bromooctadecane was purchased from Fluka (France). 4-nitro phenol and 4-nitrophenyl chloroformate were obtained from Merck (Germany) and methoxypolyethylene glycol 2,000 was purchased from Sigma-Aldrich (Germany). The phospholipids, were purchased from Lipoid (Ludwigshafen, Germany). 1,2-dipalmitoyl-sn-glycero-3-phosphoethanolamine-N-(lissamine rhodamine B sulfonyl) (DPPE-lissamine rhodamine B) were obtained from Avanti Polar Lipids (Alabaster, AL). Cholesterol, were purchased from Sigma-Aldrich (St. Louis, MO). Caelyx ${ }^{\circledR}$ was obtained from Behestan 
Darou Company (Tehran, Iran). RPMI 1640 culture medium and fetal bovine serum (FBS) were obtained from Gibco (Carlsbad, CA).

\section{Preparation and characterization of hypoxia sensitive linker (PEG-azolinker- $\left.\mathrm{C}_{18} \mathrm{H}_{37}\right)$}

Synthesis and characterization of 4,4 $4^{\mathbb{X}}$-Dihydroxyazobenzene (1a),4-((4(octadecyloxy)phenyl)diazenyl)phenol (2) and the functionalized hydroxy terminated methoxy polyethylene glycol $2000\left(\mathrm{mPEG}_{2000}\right)(\mathbf{1 b})$ are noted in the supplementary materials.

To a solution of 4-((4-(octadecyloxy)phenyl)diazenyl)phenol $(0.32 \mathrm{mmol}, 150 \mathrm{mg})$ in chloroform $(5 \mathrm{ml})$, functionalized $\mathrm{mPEG}_{2000}$ was added and refluxed. After 24 hours of refluxing, the solvent was evaporated under vacuum condition and a mixture of ethanol/water $(15 \mathrm{ml} / 5 \mathrm{ml})$ was added to obtain a suspension.

The suspension was centrifuged at $250 \mathrm{rpm}$ for 15 minutes. The supernatant was separated and the solvent (ethanol/water) evaporated under reduced pressure. The yellow residue was dissolved in chloroform and washed with water three times to remove the (none) un-reacted functionalized $\mathrm{mPEG}_{2000}$. After solvent (chloroform) removing under reduced pressure the resulted yellow linker was washed three times by cold ether.

PEG-azolinker- $\mathrm{C}_{18} \mathrm{H}_{37}$; yellow solid; mp 56 - 59 هC; ${ }^{1} \mathrm{H}$ NMR (300MHz, DMSO-d6) $\delta(\mathrm{ppm})$ : 0.86 (t), $1.19-$ $1.42(\mathrm{~m}), 1.76(\mathrm{p}), 3.53-3.66(\mathrm{~m}), 3.72-3.77(\mathrm{~m}), 4.13(\mathrm{t}), 4.31-4.40(\mathrm{~m}), 6.88-6.95(\mathrm{~m}), 7.11-7.16$ $(\mathrm{m}), 7.46-7.49(\mathrm{~m}), 7.89-7.94(\mathrm{~m}) . \mathrm{IR}(\mathrm{KBr}): \mathrm{u}_{\max }\left(\mathrm{cm}^{-1}\right) 1107(\mathrm{C}-\mathrm{O}), 1601(\mathrm{~N}=\mathrm{N}), 1760(\mathrm{C}=0)$.

\section{Cationic liposome (Cat-lip) preparation}

Lipids consist of DOTAP, DOPC and Cholesterol dissolved in chloroform at a molar ratio of 30,35,35 respectively (Total lipid: $50 \mathrm{mM}$ ) were mixed and the thin film was prepared after removing solvent using a rotary evaporator and subsequent freeze-drying. For the preparation of liposome with the final volume of $1 \mathrm{~mL}$, the lipid film was dissolved in pre-heated absolute ethanol at $65^{\circ} \mathrm{C}$. Then, a pre-heated ammonium sulfate solution $(250 \mathrm{mM})$ at $65^{\circ} \mathrm{C}$ was injected with a Hamilton syringe into the solution that was concurrently shaken on the vortex. Lipoid colloidal suspension then extruded through polycarbonate membranes of $200 \mathrm{~nm}, 100 \mathrm{~nm}$, and $50 \mathrm{~nm}$ sequentially. To remove the free ammonium sulfate and provide the conjugation medium, liposomes were then dialyzed against dextrose histidine $10 \mathrm{mM},(\mathrm{pH}$ 6.5). Liposomes with encapsulated ammonium sulfate were then incubated with Dox solution (1 mg Dox per $12 \mu \mathrm{mol}$ of total lipid) at $65 \circ \mathrm{C}$ for $60 \mathrm{~min}$, cooled to room temperature. In order to remove free Dox, liposomes were dialyzed against dextrose histidine (10mM, $\mathrm{pH} 6.5)$.

\section{Post-insertion and characterization of PEGylated Catt-lip containing azo linker (PEG-Azo-Cat-lip)}

The process of post-insertion was performed at $65^{\circ} \mathrm{C}$ for $1 \mathrm{~h}$. To do this, the amount of $5 \%$ of PEGazolinker- $\mathrm{C}_{18} \mathrm{H}_{37}(2.5 \mathrm{mM}, \mathrm{MW}=2508 \mathrm{~g})$ was calculated and added to a certain volume of the cationic liposome. After $1 \mathrm{~h}$ incubation at $65^{\circ} \mathrm{C}$, the formulation was dialysis against dextrose histidine $(10 \mathrm{mM}$, 
$\mathrm{pH}$ 6.5) and the amounts of Dox in liposomes was measured using fluorimetry (Perkin-Elmer LS-45, US). (excitation:emission 490:585). Particle size and zeta potential of liposomes were measured by Horiba SZ100 (HORIBA, Kyoto, Japan).

\section{Release study}

Release study was performed using dialysis tubing 12-14 kDa MWCO. The release medium was PBS (pH 7.4) supplemented with $100 \mu \mathrm{M}$ NADPH and $2 \mathrm{mg} / \mathrm{mL}$ rat liver microsome [18]. Rat liver microsomes were extracted using the simple method by Kamath et. al., which contain reducing enzymes that help to break down azo-linker [19]. One milliliter of the formulations was placed in the 12-14 molecular weight cut-off dialysis tubing, and the tube was immersed in the $50 \mathrm{~mL}$ of the release medium. The hypoxic condition was established by nitrogen gas flow, and normoxic condition was established using airflow. At certain time intervals, one milliliter of the medium was removed and replaced by fresh medium. The Dox concentration was calculated using fluorimetry (Perkin-Elmer LS-45) (Ex:Em 490:585). The cumulative release was then calculated.

\section{Cytotoxicity}

Cytotoxicity study was performed into different conditions, hypoxic and normoxic conditions, and studied using MTT assay. First, C26 cells were seeded at the density of 5000 cells per each well at 96 -well plates. Then one of 96 -well plates was placed at the humidified incubator at $37^{\circ} \mathrm{C}, 5 \% \mathrm{CO} 2$ to induce the normoxic conditions. Another 96 -well plate was placed at a hypoxic chamber and then the chamber was placed at a humidified incubator. The gas mixture ( $1 \% 02,5 \% \mathrm{CO} 2$, balanced with $\mathrm{N} 2)$ was flowed every 2 $\mathrm{h}$ for $5 \mathrm{~min}$ to assure the maintenance of the hypoxic condition. After overnight incubation, the cells were subjected to different concentrations of the formulations for $24 \mathrm{~h}$ and then MTT was added to each well and the cells incubated for the $4 \mathrm{~h}$ and after dissolving formazan crystal in DMSO the absorbance were measured at $570 \mathrm{~nm}$ using Stat-Fax 2100 microplate reader (Awareness Technology Inc. /USA). The IC ${ }_{50}$ values were calculated by CalcuSyn version 2 software (BIOSOFT, UK).

\section{Animals}

Female BALB/c mice (4-8 weeks old) were purchased from Pasteur institute of Iran (Tehran, Iran). Animals were kept at the $12 \mathrm{~h}: 12 \mathrm{~h}$ light: dark cycle in the animal house. All animals had free access to food and water. All experimental protocols were approved by the local institutional committee for animal ethics (ethical number: 982901) and were performed according to the international rules considering animal rights.

\section{Biodistribution}

Liposomes were prepared using DPPE-lissamine rhodamine B (0.2\% molar ratio) as a fluorescent tracking agent. Mice were inoculated with C26 tumor cells $\left(3 \times 10^{5}\right.$ cells per mouse) in the right flank. After fourteen days post- inoculation, intravenous (i.v.) injections of the dose of $15 \mathrm{mg} / \mathrm{kg}$ of Cat-lip and PEG- 
Azo-Cat-lip were performed in mice $(n=3)$. At the times of $3,6,24,48,72,96$ and 120 h photographs were taken using Small Animal Imaging System (Kodak FX Pro, Eastman Kodak, Rochester, NY, USA). The excitation wavelength was $560 \mathrm{~nm}$ and the emission wavelength was $583 \mathrm{~nm}$.

The biodistribution of formulations also was evaluated using the intrinsic fluorescent of Dox. To do this, mice were inoculated with $\mathrm{C} 26$ tumor cells $\left(3 \times 10^{5}\right.$ cells per mouse) in the right flank. After fourteen days post-inoculation, mice ( $\mathrm{n}=3$ in each group) were i.v. administrated with $15 \mathrm{mg} / \mathrm{kg}$ of Cat-lip and PEG-AzoCat-lip. At certain time intervals ( 3,24 and $48 \mathrm{~h}$ post-injection) mice were anesthetized using the mixture of ketamine and xylazine. The blood samples were directly collected from the heart and then, major organs including, the heart, lung, liver, spleen, kidney, and the tumor were removed, weighed and homogenized in acidic isopropyl. The fluorescent intensity of Dox was determined for each sample using Perkin-Elmar spectrofluorometer (LS-45, Perkin-Elmer, USA). The concentrations of Dox in blood and organs were calculated.

\section{Anti-tumor activity}

Mice were inoculated with C26 tumor cells $\left(3 \times 10^{5}\right.$ cells per mouse) in the right flank. A week after tumor inoculation, mice ( $n=5$ in each group) with palpable tumor size were received a single dose of $10 \mathrm{mg} / \mathrm{kg}$ (Dox) of liposomal formulations and commercially available PEGylated liposomal Dox (Caelyx ${ }^{\circledR}$ ) via vein tail injection. One group received $5 \%$ dextrose solution as the negative control. The tumor size and weights of mice were monitored over a 60-day period. Three diameters of tumors were measured using caliper and volumes of tumors were quantified (width $(\mathrm{mm}) \times$ width $(\mathrm{mm}) \times$ length $(\mathrm{mm})$ ). The survival parameters including median survival time (MST), time to reach the end (TTE), and percentage of tumor growth delay (TGD\%) were determined.

\section{Histological evaluations}

The biosafety effects of formulations were investigated on healthy mice intravenously injected by the dose of $10 \mathrm{mg} / \mathrm{kg}$ of the formulation. To do this, female BALB/c mice (4-8 weeks old, 18-22 g) were randomly divided into four groups $(n=3)$ and i.v. injected from the tail vein. 20 days post-injection, the mice were euthanized and major organs including the heart, lung, spleen, liver and kidney were undergone histological evaluations and were stained using hematoxylin \& eosin (H\&E) staining method. Then, the sections were examined using light microscopy.

\section{Statistical analysis}

Statistical analysis and graphical presentation were performed using GraphPad Prism 6.0 (GraphPad software, Inc., San Diego, CA, USA). Data were expressed as mean \pm SEM of at least three independent experiments. Student's t-test was performed for comparison between groups. Survival time was calculated by the Kaplan-Meier method and analyzed by the log-rank test. $\mathrm{P}<0.05$ was considered statistically significant. 


\section{Results And Discussion}

The elevated levels of reducing enzymes in hypoxic areas located at the core of solid tumors could be exploited for design NDDSs along with a high-oxidized moiety like azo-compounds [20]. In order to overbear the poor accessibility to the core of solid tumors, in this study, we designed and synthesized a cost-effective hypoxia sensitive azo-linker composing $\mathrm{C}_{18} \mathrm{H}_{37}$ lipophilic tail, azo-linker, and $\mathrm{PEG}_{2000}$ hydrophilic molecule. All parts of the linker were synthesized from commercially accessible compounds and inexpensive methods. Figure1 A depicts the route synthesis of the hypoxia-responsive linker. As demonstrated in figure 1B, this linker is cleaved via the processes performed in three steps via various types of reductases in an oxygen-dependent manner [21]. In brief, in the first step, 4,4囚-

Dihydroxyazobenzene was synthesized using Willstatter and Benz method [22]. Eighteen carbon alkyl chain $\left(\mathrm{C}_{18} \mathrm{H}_{37}\right)$ as the lipophilic part of the linker was conjugated to one of the hydroxyl groups of $4,4 \rrbracket-$ Dihydroxyazobenzene. Finally, functionalized hydroxyl-terminated $\mathrm{PEG}_{2000}$ was added to another hydroxyl group of 4,4区-Dihydroxyazobenzene. All intermediates and resultant linker were characterized using ${ }^{1}$ HNMR and FTIR (see figure 2 and 3 ).

In the next step, the hypoxia-sensitive PEG-azo- $\mathrm{C}_{18} \mathrm{H}_{37}$ was post-inserted into the Cat-lip to reduce its positive charges and confer more circulation time characteristics. The size and zeta potential results are demonstrated in table 1, in which Cat-lip was $120 \mathrm{~nm}$ in size with $18.4 \mathrm{mV}$ zeta-potential. After postinsertion, the size and zeta-potential of the resultant PEG-Azo-Cat-lip had $139 \mathrm{~nm}$ and $6.1 \mathrm{mV}$, respectively. This change in size and zeta could be the result of successful post-insertion [23]. The size measurement of the liposomes also indicated that they were in an appropriate size for tumor accumulation via EPR effects ( 100-150 nm) [24].

The cytotoxicity effects of the formulations were performed in normoxic and hypoxic conditions to compare the potencies of the liposomes [25]. The hypoxia was induced through culturing the cells in the hypoxia chamber with gas mixture flow consist of $1 \% 02,5 \% \mathrm{CO} 2$, balanced with N2. Figure 4 shows the comparison of the cytotoxicity effects of the formulation in hypoxic and normoxic conditions. As demonstrated, there were no significant differences in cytotoxic effects of Cat-lip, Caelyx ${ }^{\circledR}$, and free Dox between normoxic and hypoxic conditions. The free Dox was freely pass through the cell membrane in both conditions and induce its cytotoxic effects. Due to the high positive charge, Cat-lip also fused with the cell membrane, hence exert its cytotoxicity. In contrast, the PEGylated liposomal Dox (Caelyx ${ }^{\circledR}$ ), had the lowest levels of cytotoxicity among the formulations in both hypoxic and normoxic conditions. In the case of the PEG-Azo-Cat-lip, however, there was a difference between hypoxia and normoxia, in which higher levels of cytotoxicity were observed in the hypoxic conditions. This could be due to the detachment of the PEG molecule via hypoxia-sensitive linker and exposure of the positively charged cationic liposomes to the cells. Moreover, these results are in agreement with the results of Joshi et. al. in which they used hypoxia-sensitive linker in micelle for co-delivery of siRNA and chemotherapeutics [26].

The release study of PEG-Azo-Cat-lip was performed in hypoxic and normoxic conditions. The release of Cat-lip in the normoxic and hypoxic conditions was about 30\% (figure 5A and B). However, in the case of 
PEG-Azo-Cat-lip the release was less, about $6 \%$ in the normoxic and $8 \%$ in hypoxic conditions (figure $5 \mathrm{~A}, \mathrm{~B}$ and $\mathrm{C}$ ). As demonstrated in figure 4A, the release profile of the PEG-Azo-Cat-lip in hypoxia was more than normoxia and increased over time. The detachment of the PEG molecule and destabilizing the liposomal formulation would be a reason for this [26]; however, this difference was not statistically significant. Figure 5D showed the changes in the zeta-potentials of the PEG-Azo-Cat-lip at the time zero $(\mathrm{h})$ and after $24 \mathrm{~h}$ in hypoxic and normoxic conditions. In normoxia, there was no significant change in the zetapotential of PEG-Azo-Cat-lip between time 0 and $24 \mathrm{~h}$. However, in hypoxic conditions, there was a dramatic decrease in the zeta-potential of PEG-Azo-Cat-lip and there was a significant difference between zeta-potential of PEG-Azo-Cat-lip in hypoxic and normoxic condition at time $24 \mathrm{~h}$. This could be due to the detachment of PEG molecule and exposure of the positive charges which electrostatically absorb enzymes that exist in the rat-liver microsome. However, at the tumor microenvironment, this exposure also could occur for the negatively charged cell membranes, which results in high rates of cellular uptake and consequent cytotoxic effects.

The biodistribution of the Cat-lip and PEG-Azo-Cat-lip was performed in mice bearing C26 tumor models. The formulations were prepared using DPPE-lissamine rhodamine $B$, which is detectable in animal imaging device [27]. Figure 6 showed the biodistribution of the formulations, after i.v. injection of the 10 $\mathrm{mg} / \mathrm{kg}$ via tail vein during $120 \mathrm{~h}$. As qualitatively shown, the accumulation of the PEG-Azo-Cat-lip was more than Cat-lip over the $120 \mathrm{~h}$ of the study, which indicated the efficacy of the PEG-Azo-Cat-lip formulation in comparison with Cat-lip.

The biodistribution of the Cat-lip and PEG-Azo-Cat-lip was also investigated in tumors and organs including, the heart, kidney, spleen, liver and lung using spectrofluorimetry. Figure 7 shows the plasma concentrations of the formulation during the 48 hours of the study. As demonstrated PEG-Azo-Cat-lip had higher concentrations than Cat-lip in all times of the study, which is due to the PEGylation and negative charges of this formulation. PEGylation confers the formulation steric hindrance, and negative charge make the formulation to avoid RES [28]. The plasma concentration of the Cat-lip was lower than PEGAzo-Cat-lip over the times of the study, in which at the time 48 the Dox concentration has reached zero and cleared from the blood. However, in the case of PEG-Azo-Cat-lip the concentration of Dox was higher than Cat-lip, and also it was not reached to the zero at the time $48 \mathrm{~h}$. The mechanism lies behind the more circulation time for PEG-Azo-Cat-lip could be a polymer coating with PEG molecule which resulted in the reduced recognition by the RES [29]. The rapid clearance of the Cat-lip from the blood was consistent with the release data that was more than $30 \%$ over the $24 \mathrm{~h}$ and was considerably higher than PEG-Azo-Cat-lip.

The anti-tumor efficiencies of the liposomes were also studied on mice bearing C26 tumor models and monitored over a period of two months and compared with Caelyx ${ }^{\circledR}$ [23]. It was shown that during the development of C26 tumor model in mice there were two peaks of hypoxia, first at early stages of tumor formation and second peak during formation of necrotic areas due the fast-growing tumor cells [30]. Here we have injected the formulations at the early stage of tumor formation where the hypoxic microenvironment was taking place. As demonstrated in figure 9A, all liposomal formulations did not have dramatic effects on the animal weight, which indicated the prepared formulations were as efficient 
as Caelyx ${ }^{\circledR}$. Figure 9B showed the results of tumor volume. The results have showed the efficiency of the PEG-Azo-Cat-lip compared to the Caelyx ${ }^{\circledR}$ and Cat-lip. Figure $9 \mathrm{C}$ depicted the survival plot of all liposomal formulations compared to the PBS group. All formulations have increased the survival compared to the PBS group. Again, the results indicated the efficiency of the PEG-Az-Cat-lip compared to the Caelyx ${ }^{\circledR}$ and Cat-lip. The main indicators of survival study, including MST, TTE, and \%TGD were summarized in Table 2. Data showed that the greatest MST and \%TGD were seen in the mice treated with PEG-Azo-Cat-lip (MST= 54 days, \%TGD = 79.2). Treatment with PEG-Azo-Cat-lip increased TTE and resulted in effective anti-tumor activity with $79.2 \%$ TGD.

Histological evaluations of the formulations were shown in figure 10. The results indicated the signs of inflammation and tissue rearrangement of Cat-lip treated mice in the heart, lung, liver, kidney and spleen. The rapid release of Dox from Cat-lip as indicated by release study and plasma concentration could be responsible for the toxicity in these major organs, which leads to show similar behavior to the free Dox [31, 32]. The presence of DOTAP, a cationic lipid with positive charge, also could be a reason for such toxicity, as demonstrated by in vitro gene delivery studies [33,34]. The effects of PEG-Azo-Cat-lip on the tissue, regarding the biosafety of the formulation was similar to the Caelyx ${ }^{\circledR}$ that indicated the effect of PEGylation on the safety of the PEG-Azo-Cat-lip. as comparable as commercially available form of PEGylated liposomal Dox.

\section{Conclusion}

In this study, we have developed a hypoxia-sensitive formulation, based on a highly oxidized azo compound. At first, Dox cationic liposome was prepared and PEG-Azo- $\mathrm{C}_{18} \mathrm{H}_{37}$ compound was synthesized using commercially accessible compounds through inexpensive methods. Desired formulation of PEG-Azo-Cat-lip was yield after post-insertion of the PEG-Azo- $\mathrm{C}_{18} \mathrm{H}_{37}$ into the cationic liposome. This azo-linker probably would be broken in response to the hypoxic tumor environment. The cleavage resulted in the determent of PEG molecule and exposure of the positive charges on the surface of the liposome. The results of in vitro and in vivo studies indicated the efficacy of PEG-Azo-Cat-lip formulation. It is suggested to perform the efficacy of this hypoxia-sensitive formulation on different mice tumor models and also on nude mice.

\section{Declarations}

\section{Acknowledgements}

Research reported in this publication was supported by Elite Researcher Grant Committee under award number [982901] from the National Institute for Medical Research Development (NIMAD), Tehran, Iran.

\section{Declaration of interests}


The authors declare that they have no known competing financial interests or personal relationships that could have appeared to influence the work reported in this paper.

\section{References}

1. Rosenblum D, Joshi N, Tao W, et al (2018) Progress and challenges towards targeted delivery of cancer therapeutics. Nat Commun 9:1410

2. Golombek SK, May J-N, Theek B, et al (2018) Tumor targeting via EPR: strategies to enhance patient responses. Adv Drug Deliv Rev

3. Mitchell MJ, Jain RK, Langer R (2017) Engineering and physical sciences in oncology: challenges and opportunities. Nat Rev Cancer 17:659

4. Zhao W, Zhuang S, Qi X-R (2011) Comparative study of the in vitro and in vivo characteristics of cationic and neutral liposomes. Int J Nanomedicine 6:3087

5. Deshpande PP, Biswas S, Torchilin VP (2013) Current trends in the use of liposomes for tumor targeting. Nanomedicine 8:1509-1528

6. Muhamad N, Plengsuriyakarn T, Na-Bangchang K (2018) Application of active targeting nanoparticle delivery system for chemotherapeutic drugs and traditional/herbal medicines in cancer therapy: a systematic review. Int J Nanomedicine 13:3921

7. Yoo J, Park C, Yi G, et al (2019) Active targeting strategies using biological ligands for nanoparticle drug delivery systems. Cancers (Basel) 11:640

8. Mashreghi M, Azarpara H, Bazaz MR, et al (2018) Angiogenesis biomarkers and their targeting ligands as potential targets for tumor angiogenesis. J Cell Physiol 233:2949-2965

9. Mura S, Nicolas J, Couvreur P (2013) Stimuli-responsive nanocarriers for drug delivery. Nat Mater 12:991-1003

10. Ganta S, Devalapally H, Shahiwala A, Amiji M (2008) A review of stimuli-responsive nanocarriers for drug and gene delivery. J Control release 126:187-204

11. Liu M, Du H, Zhang W, Zhai G (2017) Internal stimuli-responsive nanocarriers for drug delivery: Design strategies and applications. Mater Sci Eng C 71:1267-1280

12. Hanahan D, Weinberg RA (2011) Hallmarks of cancer: the next generation. Cell 144:646-674

13. Rajabi M, Mousa SA (2017) The role of angiogenesis in cancer treatment. Biomedicines 5:34

14. Chen L, Endler A, Shibasaki F (2009) Hypoxia and angiogenesis: regulation of hypoxia-inducible factors via novel binding factors. Exp Mol Med 41:849-857 
15. Zhou Y, Maiti M, Sharma A, et al (2018) Azo-based small molecular hypoxia responsive theranostic for tumor-specific imaging and therapy. J Control Release 288:14-22

16. Maleki MF, Jafari A, Mirhadi E, et al (2019) Endogenous Stimuli-Responsive Linkers in Nanoliposomal Systems for Cancer Drug Targeting. Int J Pharm 118716

17. Kiyose K, Hanaoka K, Oushiki D, et al (2010) Hypoxia-sensitive fluorescent probes for in vivo realtime fluorescence imaging of acute ischemia. J Am Chem Soc 132:15846-15848

18. Kulkarni P, Haldar MK, Katti P, et al (2016) Hypoxia responsive, tumor penetrating lipid nanoparticles for delivery of chemotherapeutics to pancreatic cancer cell spheroids. Bioconjug Chem $27: 1830-1838$

19. Kamath SA, Kummerow FA, Narayan KA (1971) A simple procedure for the isolation of rat liver microsomes. Febs Lett 17:90-92

20. Wang Y, Shang W, Niu M, et al (2019) Hypoxia-active nanoparticles used in tumor theranostic. Int J Nanomedicine 14:3705

21. Zbaida S, Levine WG (1991) A novel application of cyclic voltammetry for direct investigation of metabolic intermediates in microsomal azo reduction. Chem Res Toxicol 4:82-88

22. Willstätter R, Benz M (1906) Zur kenntniss der Azophenole. Berichte der Dtsch Chem Gesellschaft 39:3492-3503

23. Mashreghi M, Zamani P, Moosavian SA, Jaafari MR (2020) Anti-Epcam Aptamer (Syl3c)Functionalized Liposome for Targeted Delivery Of Doxorubicin: In Vitro And In Vivo Antitumor Studies in Mice Bearing C26 Colon Carcinoma. Nanoscale Res Lett 15:101. https://doi.org/10.1186/s11671-02003334-9

24. Karimi M, Gheybi F, Zamani P, et al (2020) Preparation and characterization of stable nanoliposomal formulations of Curcumin with high loading efficacy: In vitro and in vivo anti-tumor study. Int J Pharm 119211

25. Brownlee WJ, Seib FP (2018) Impact of the hypoxic phenotype on the uptake and efflux of nanoparticles by human breast cancer cells. Sci Rep 8:1-11

26. Joshi U, Filipczak N, Khan MM, et al (2020) Hypoxia-sensitive micellar nanoparticles for co-delivery of siRNA and chemotherapeutics to overcome multi-drug resistance in tumor cells. Int J Pharm 590:119915

27. Hamedinasab H, Rezayan AH, Mellat M, et al (2020) Development of chitosan-coated liposome for pulmonary delivery of N-acetylcysteine. Int J Biol Macromol 156:1455-1463 
28. Suk JS, Xu Q, Kim N, et al (2016) PEGylation as a strategy for improving nanoparticle-based drug and gene delivery. Adv Drug Deliv Rev 99:28-51

29. Bai S, Gupta V, Ahsan F (2009) Cationic liposomes as carriers for aerosolized formulations of an anionic drug: safety and efficacy study. Eur J Pharm Sci 38:165-171

30. Kiraga $\measuredangle$, Cheda $\measuredangle$, Taciak $B$, et al (2018) Changes in hypoxia level of CT26 tumors during various stages of development and comparing different methods of hypoxia determination. PLoS One 13:e0206706

31. Tacar O, Sriamornsak P, Dass CR (2013) Doxorubicin: an update on anticancer molecular action, toxicity and novel drug delivery systems. J Pharm Pharmacol 65:157-170

32. Wang L, Chen Q, Qi H, et al (2016) Doxorubicin-induced systemic inflammation is driven by upregulation of toll-like receptor TLR4 and endotoxin leakage. Cancer Res 76:6631-6642

33. Filion MC, Phillips NC (1997) Toxicity and immunomodulatory activity of liposomal vectors formulated with cationic lipids toward immune effector cells. Biochim Biophys Acta (BBA)Biomembranes 1329:345-356

34. Betker JL, Anchordoquy TJ (2015) Relating toxicity to transfection: using sphingosine to maintain prolonged expression in vitro. Mol Pharm 12:264-273

\section{Tables}

Table 1. The results of characterization of size and zeta-potentials of the formulation using DLS.

\begin{tabular}{lllll} 
Formulation & Z-average $(\mathrm{nm})$ & Zeta-potential $(\mathrm{mV})$ & $P P^{P}$ & EE\% \\
\hline Cat-lip & $120 \pm 1$ & $18.4 \pm 1$ & $0.3 \pm 0.03$ & 90.3 \\
\hline PEG-Azo-Cat-lip & $139 \pm 3$ & $6.1 \pm 2$ & $0.2 \pm 0.05$ & 90.0
\end{tabular}

a polydispersity index, ${ }^{b}$ Encapsulation efficiency (\%). The data are presented as mean \pm SD.

Table 2. The survival parameters for mice $(n=5)$ receiving different formulations. 


\begin{tabular}{lllll} 
& PBS & Cat-lip & Caelyx $^{\circledR}$ & PEG-Azo-Cat-lip \\
\hline$T T E^{a} \pm S D$ & $27.9 \pm 3.3$ & $41.1 \pm 5.1$ & $48.3 \pm 4.3$ & $51.6 \pm 3.0$ \\
\hline$T G D(\%)^{b}$ & 0 & 29.8 & 46.3 & 72.0 \\
\hline$M S T^{c}$ & 30 & 39 & 49 & 50
\end{tabular}

a Time to reach end, ${ }^{b}$ percentage of tumor growth delay, ${ }^{c}$ median survival time.

\section{Figures}

A

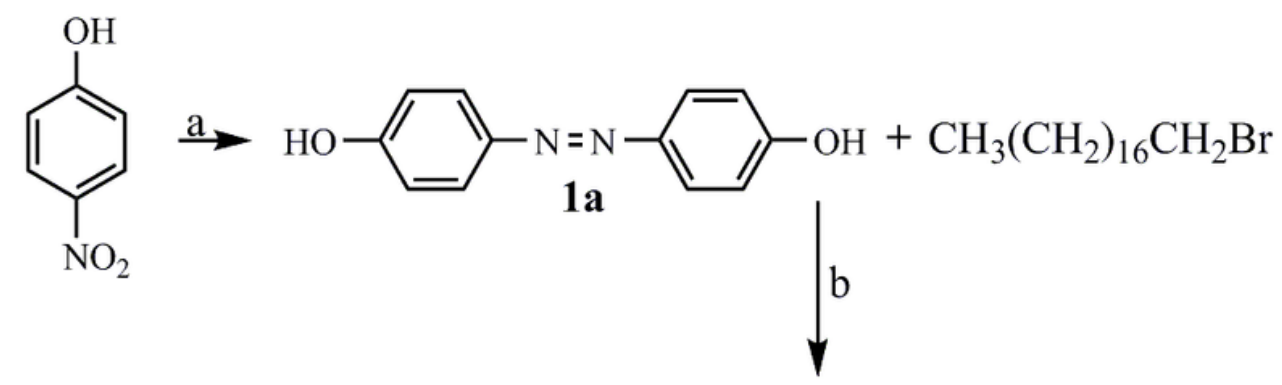<smiles>CCCCCCOc1ccc(N=Nc2ccc(O)cc2)cc1</smiles>

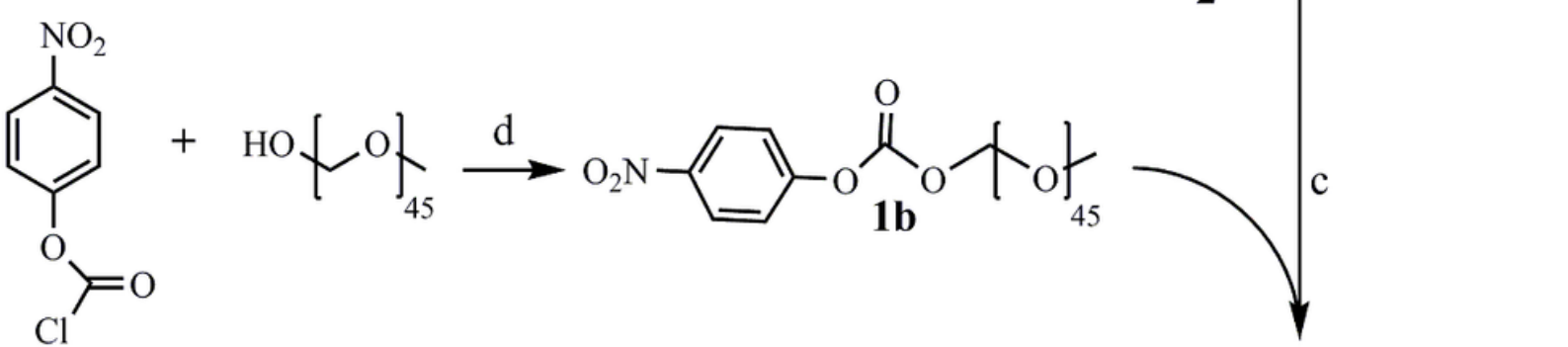<smiles>CCCCCCCCCCCCC(C)(C)OC(=O)Oc1ccc(N=Nc2ccc(OC(C)(C)C)cc2)cc1</smiles>

Hypoxia

Hydrophilic section responsive Lipophilic section

B azo

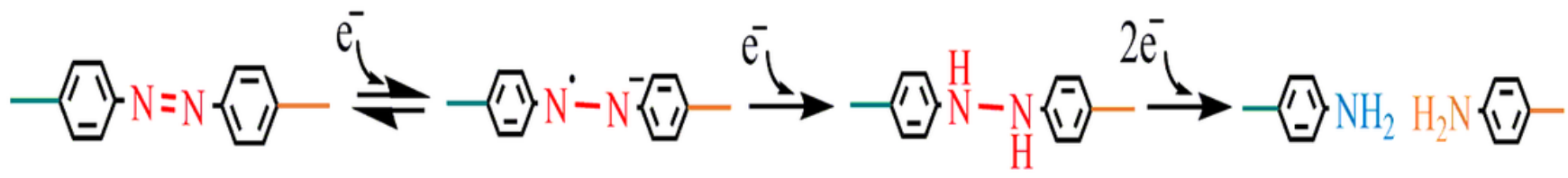


Figure 1

(A) Synthesis route of the linker; a) $\mathrm{KOH}, 220 \otimes \mathrm{c} 2 \mathrm{~h} \mathrm{~b}$ ) $\mathrm{KOH}, \mathrm{KI}, \mathrm{EtOH}$, reflux 24h c) $\mathrm{CHCl} 3$, reflux 24h d) $\mathrm{Et} 3 \mathrm{NH}, \mathrm{CH} 2 \neg \mathrm{Cl} 2$, room temperature, argon atmosphere. (B) Diazo cleavage via oxygen dependent mechanism in hypoxia areas.

A

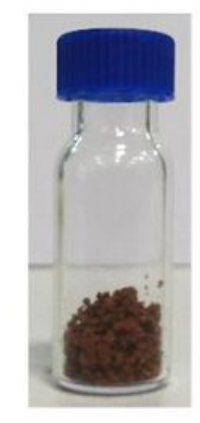

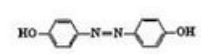

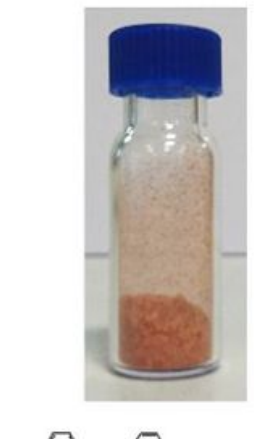

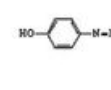

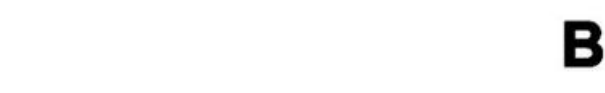

B

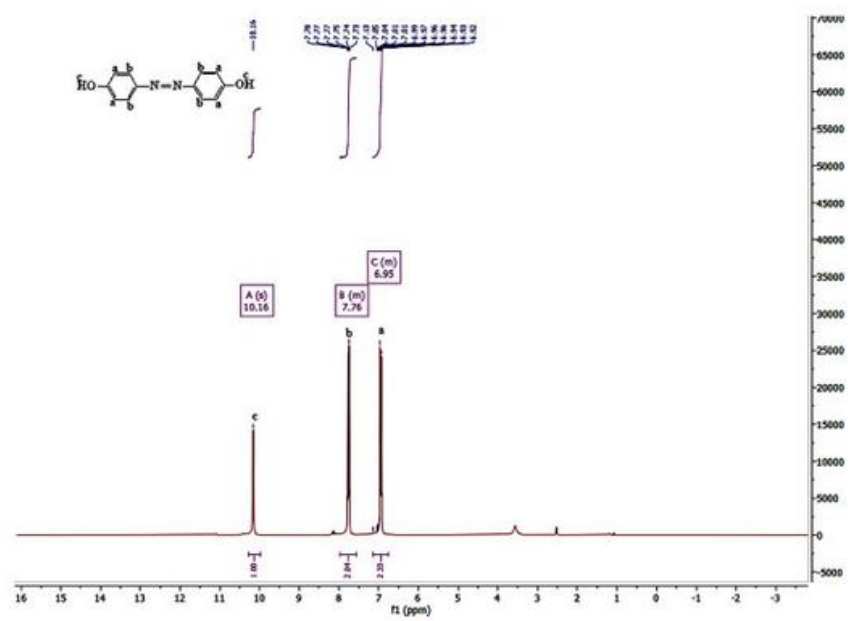

C

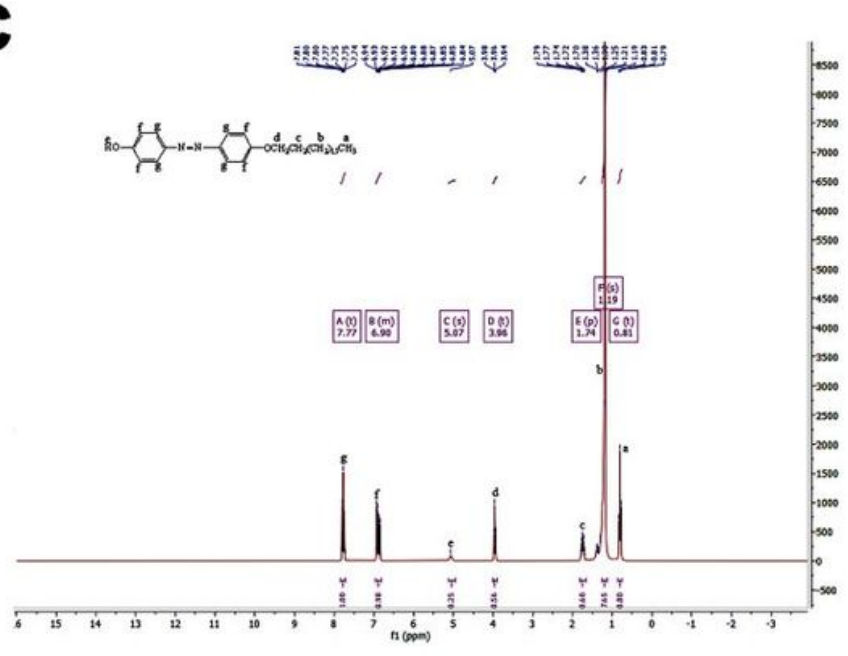

D

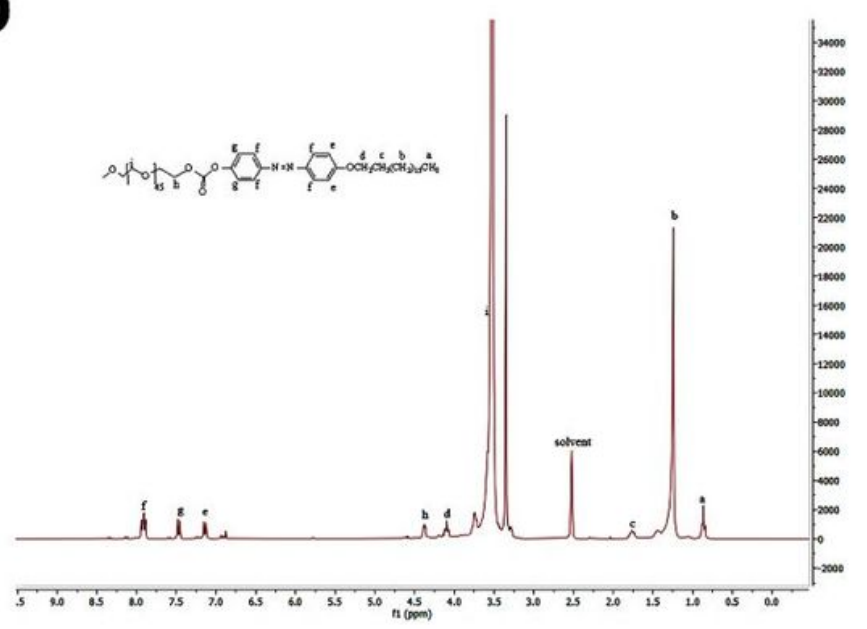

Figure 2

(A) Intermediates and the hypoxia sensitive linker (B) 1HNMR spectra of 4,4囚-Dihydroxyazobenzene (1a) (C) 4-((4-(octadecyloxy)phenyl)diazenyl)phenol (2): the peaks a ,b, c and d indicated the hydrophobic tail conjugation to the azo scaffold (1a) (D) PEG-azolinker-C18H37(3): strong peak of poly ethylene glycol (PEG)2000 appeared around 3.5 ppm (i) , the two weak triplets around 4.5 and 4 ppm are indicating $\mathrm{CH} 2-$ $O$ in PEG (h) and aliphatic tail (d) respectively. 

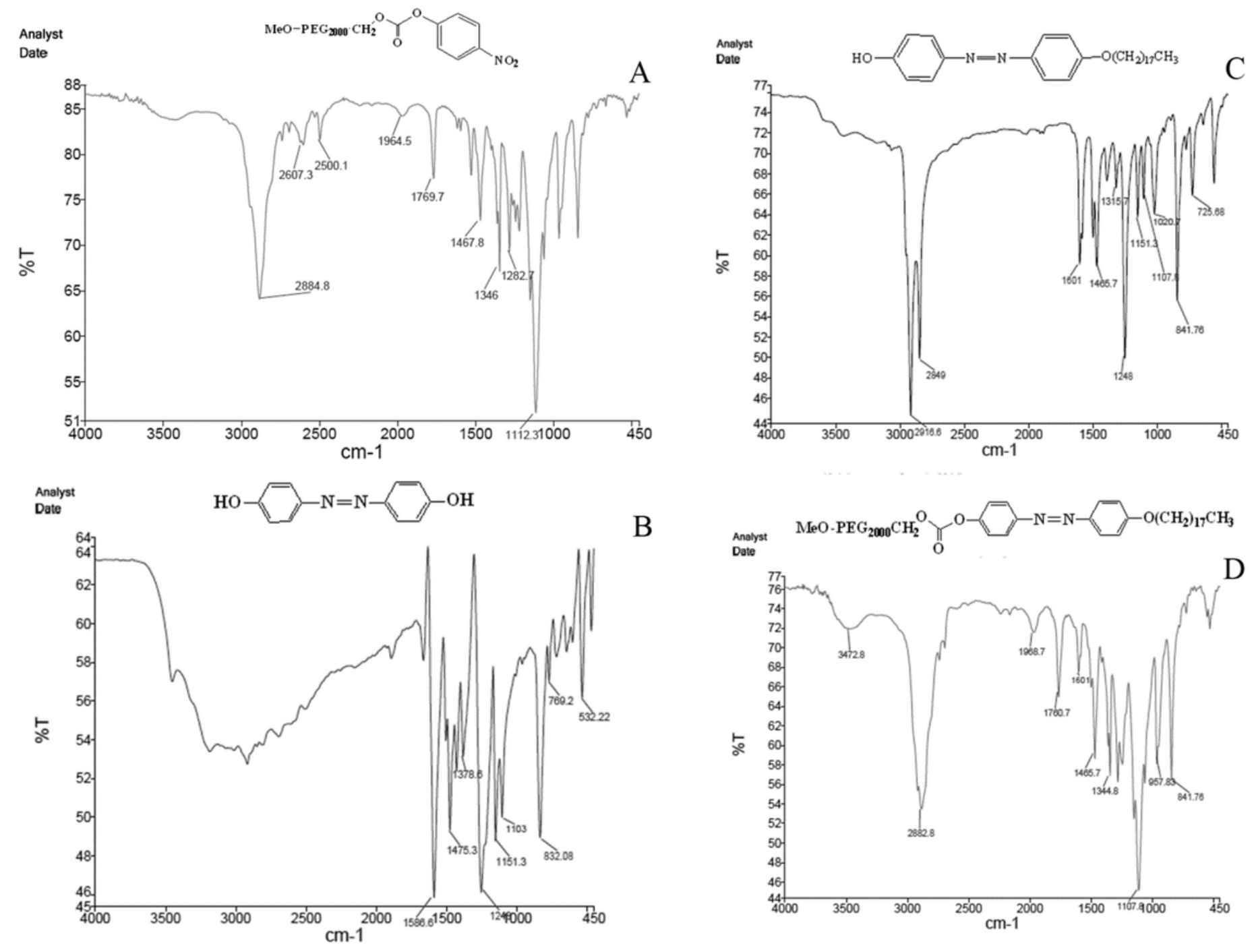

B

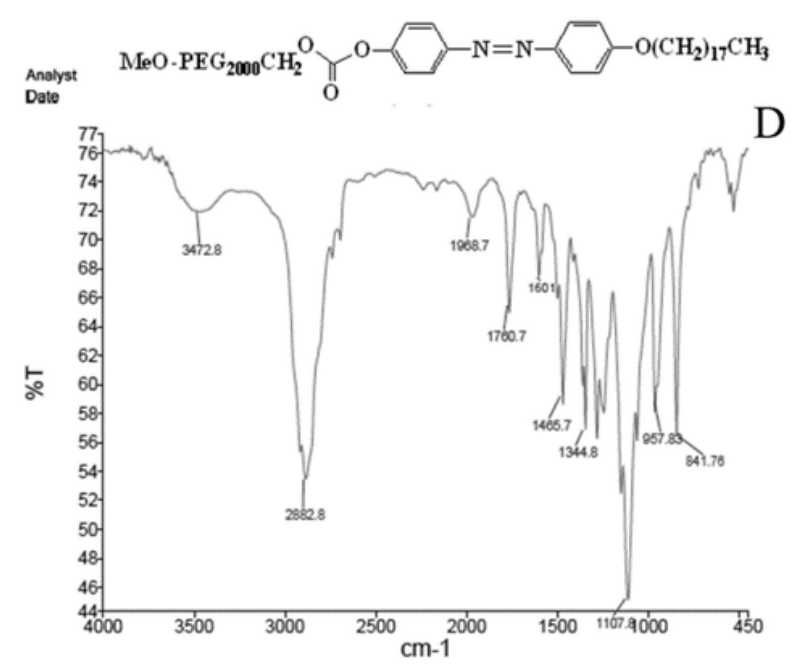

\section{Figure 3}

IR spectra of (A) functionalized hydroxyl terminated PEG2000 (1b) in which the peak in $1769 \mathrm{~cm}-1$ belongs to carbonyl group (B) 4,4囚-Dihydroxyazobenzene (1a) (C) 4,4囚-Dihydroxyazobenzene (1a) (B) 4((4-(octadecyloxy)phenyl)diazenyl)phenol (2) (D) PEG-azolinker-C18H37 (3): the shift of the $1769 \mathrm{~cm}-1$ in $1 \mathrm{~b}$ to $1760 \mathrm{~cm}-1$ originated from hydrophilic tail conjugation to the (2). 


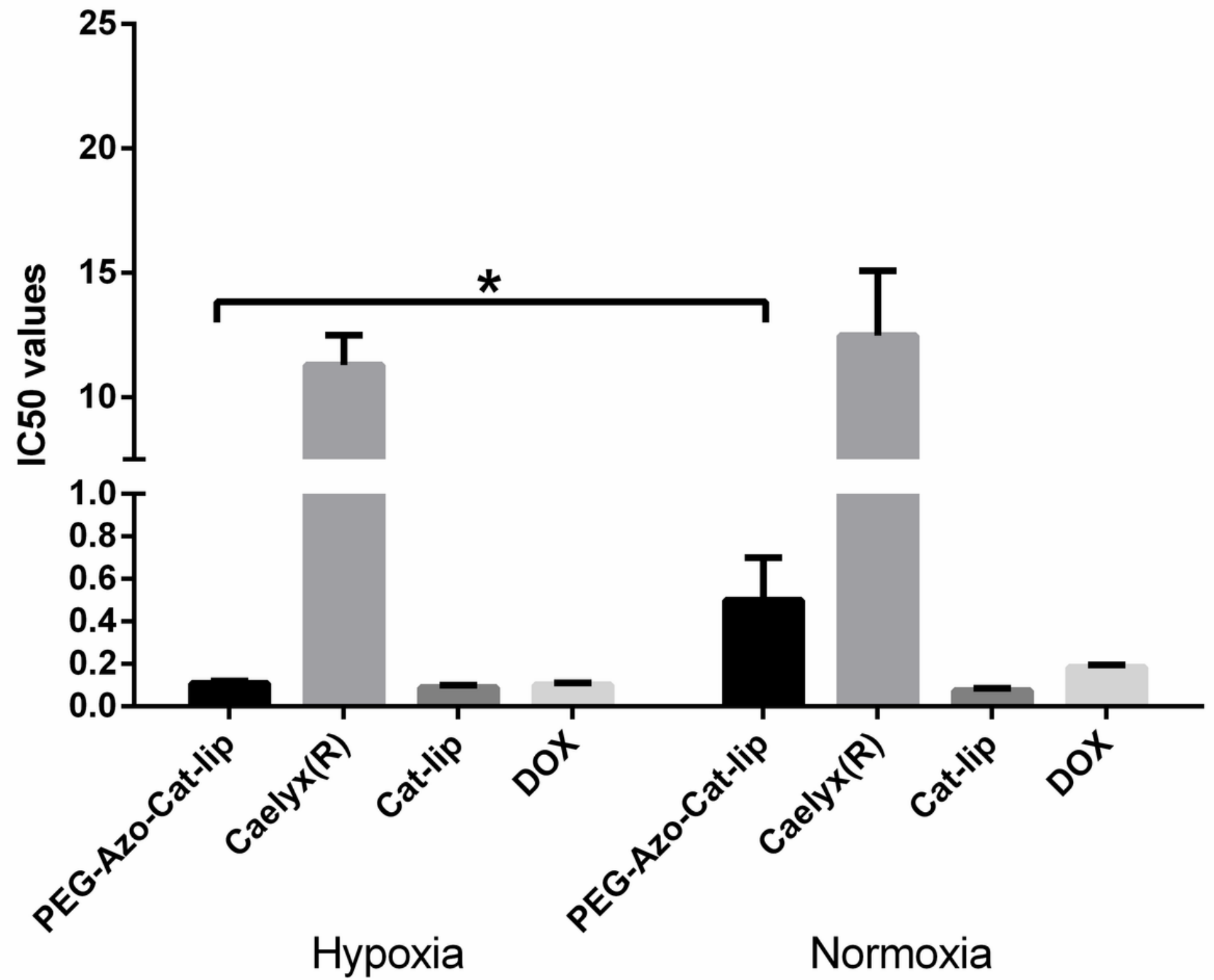

Figure 4

Cytotoxicity effects of the formulations in normoxic and hypoxic conditions. The data presented as mean \pm SD. $* p<0.05$. 

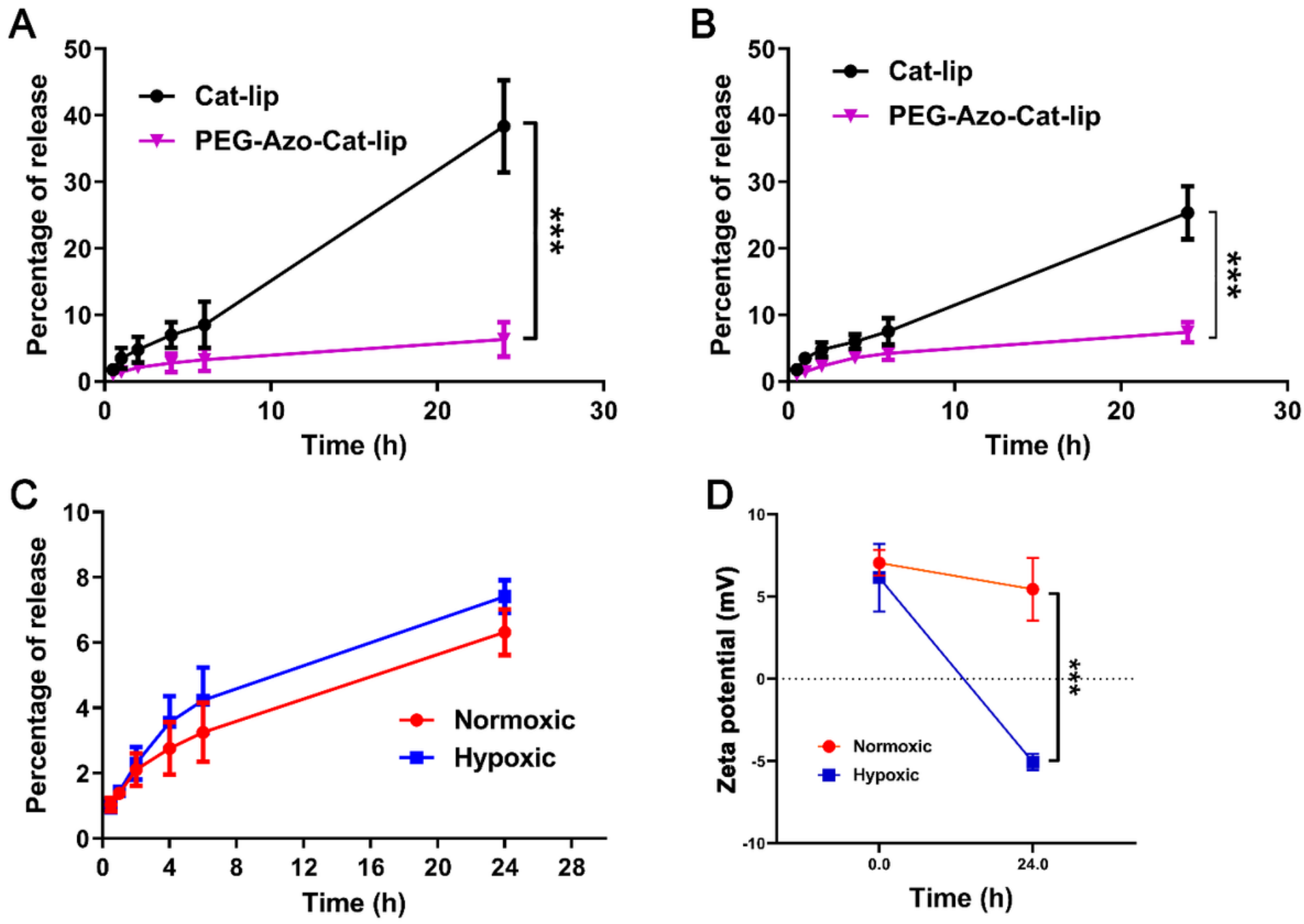

Figure 5

Release study. (A) The release of the Cat-lip and PEG-Azo-Cat-lip in normoxic condition. (B) The release of the both formulations at the hypoxic condition. (C) The release of the PEG-Azo-Cat-lip at the both hypoxic and normoxic conditions. (D) The effects of hypoxic and normoxic conditions on the zeta-potential of the PEG-Azo-Cat-lip formulation. The data presented as mean \pm SD. $* \star * ~ p<0.001$. 

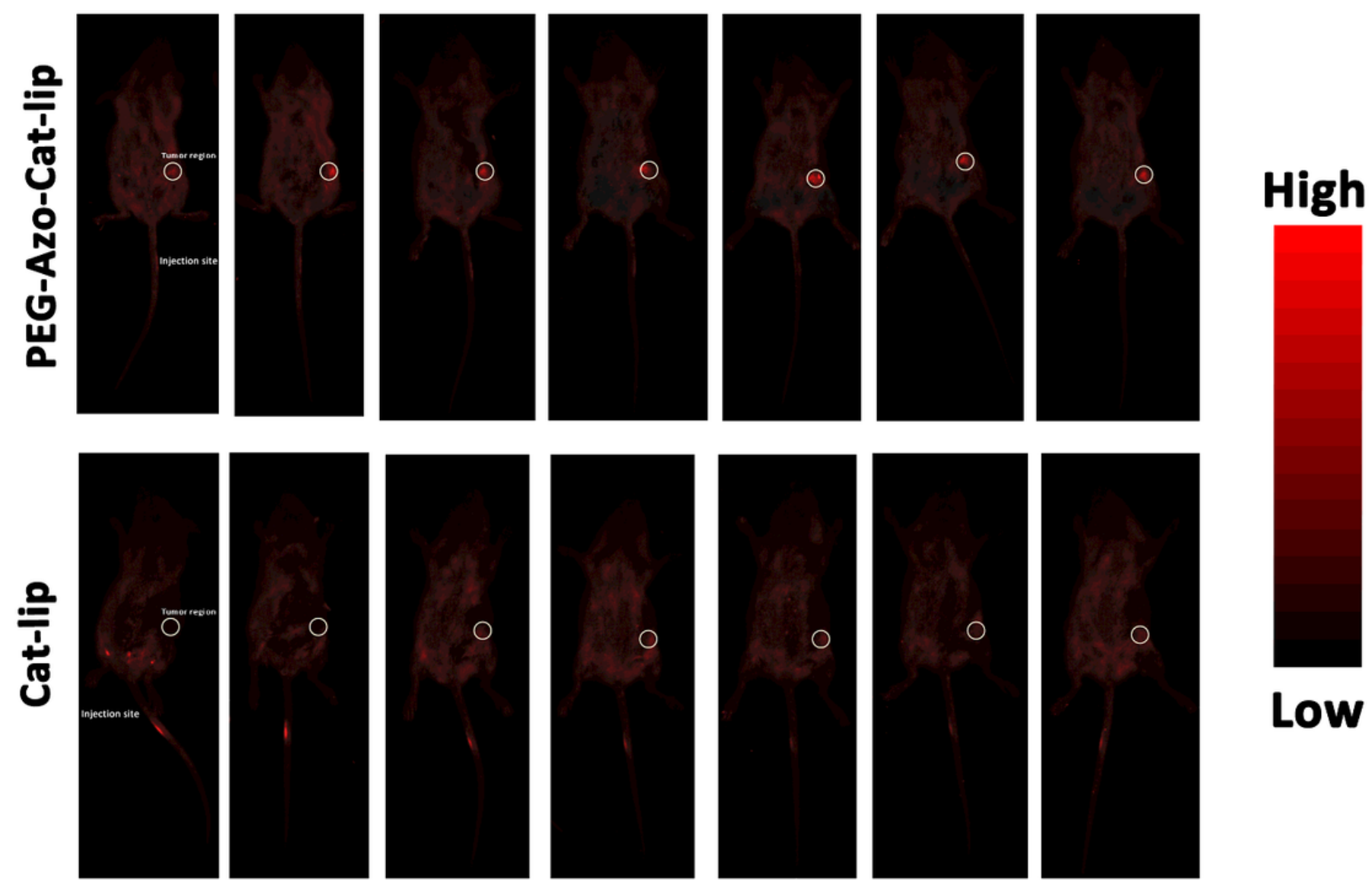

$3 h$

$6 h$

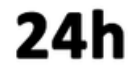

48h

$72 \mathrm{~h}$

$96 h$

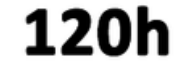

Figure 6

The results of biodistribution of the cationic liposome (Cat-lip) compared with PEGylated cationic liposome containing azo linker (PEG-Azo-Cat-lip). The formulation visualized using Kodak animal imaging though incorporation of the DPPE-lissamine rhodamine $B$ in liposomes. 


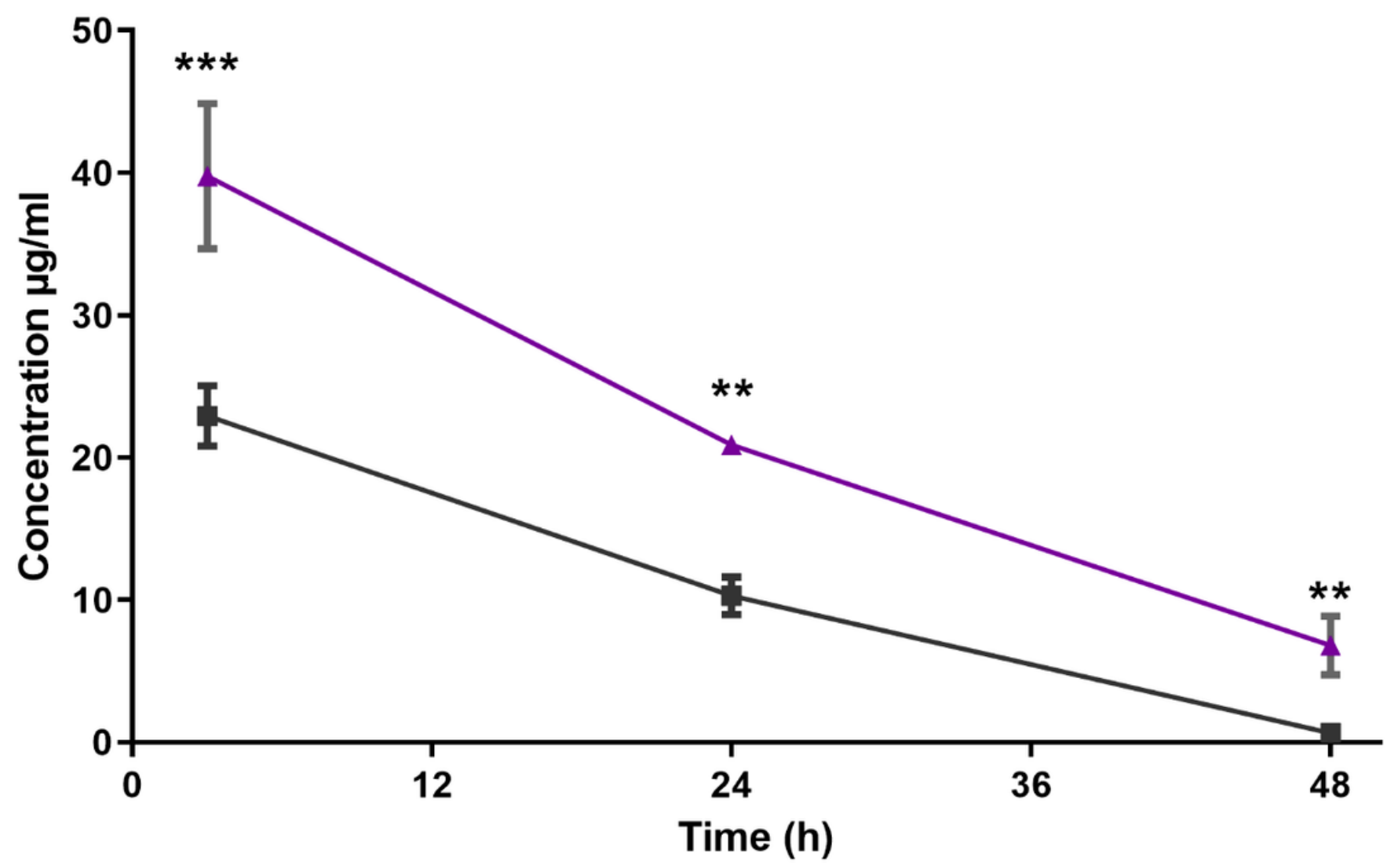

Figure 7

Plasma concentrations of the formualtions. 3, 24 and 48 hours post-injection of the $15 \mathrm{mg} / \mathrm{kg}$ i.v. injections, the concentrations of the Dox were evaluated in the plasma. The data presented as mean \pm SEM. The expriment was performed in triplicate. ${ }^{\star \star} p<0.01$ and ${ }^{\star \star \star} p<0.001$. 
Tumor

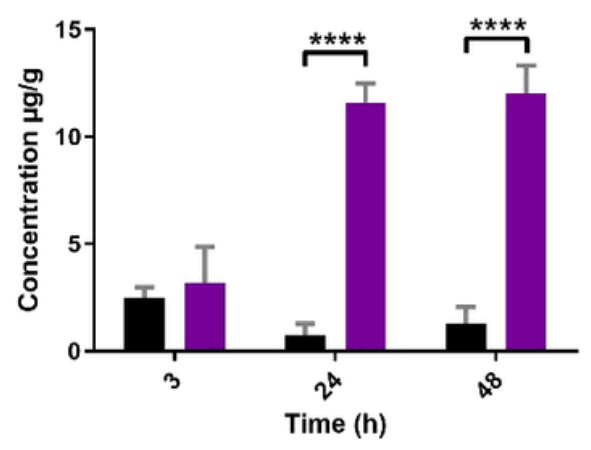

Heart

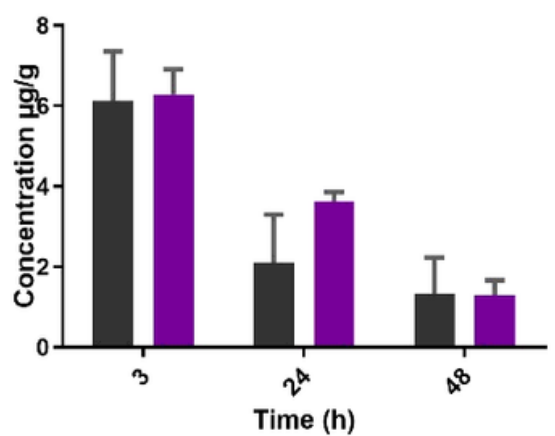

Liver

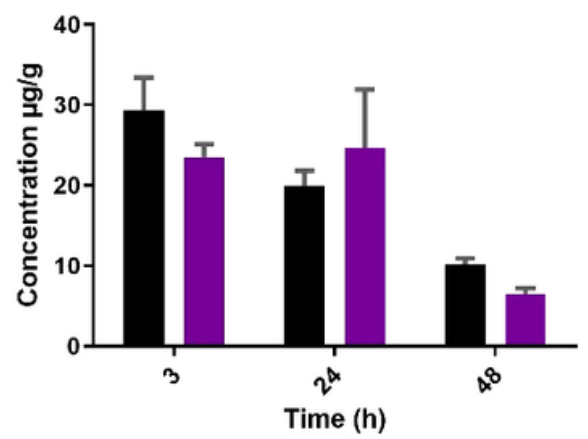

\section{Cat-lip $\quad$ PEG-Azo-Cat-lip}

Lung

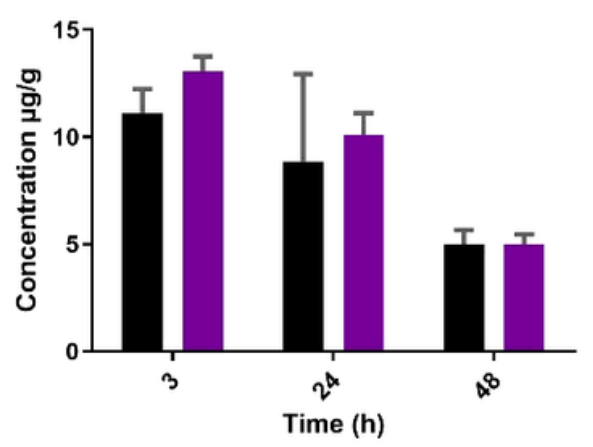

Spleen

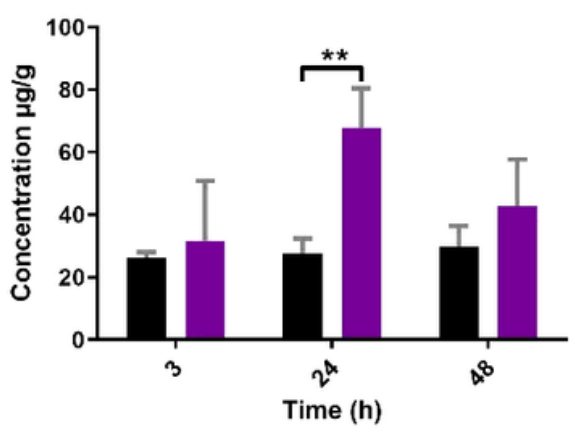

Kidney

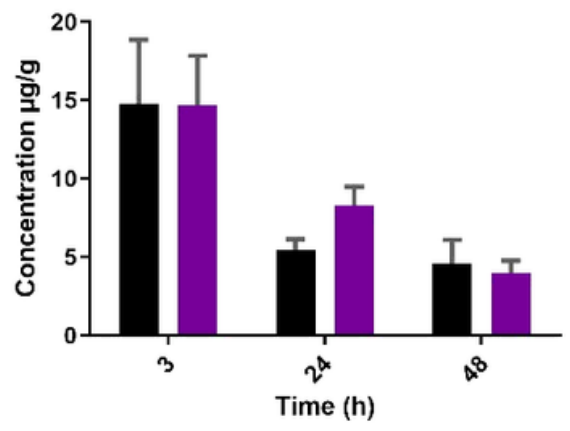

Figure 8

Dox tissue biodistribution in major organs. Concentrations of Dox of Cat-lip and PEG-Azo-Cat-lip were evaluated in six organs including tumor, heart, spleen, lung, liver and kidney using intrinsic fluorescent of Dox (Ex:Em 480:585). The data are presented as mean $\pm S D$. ${ }^{*} p<0.05$, $* \star p<0.01$, ${ }^{* \star} p<0.001, p<$ 0.0001 .
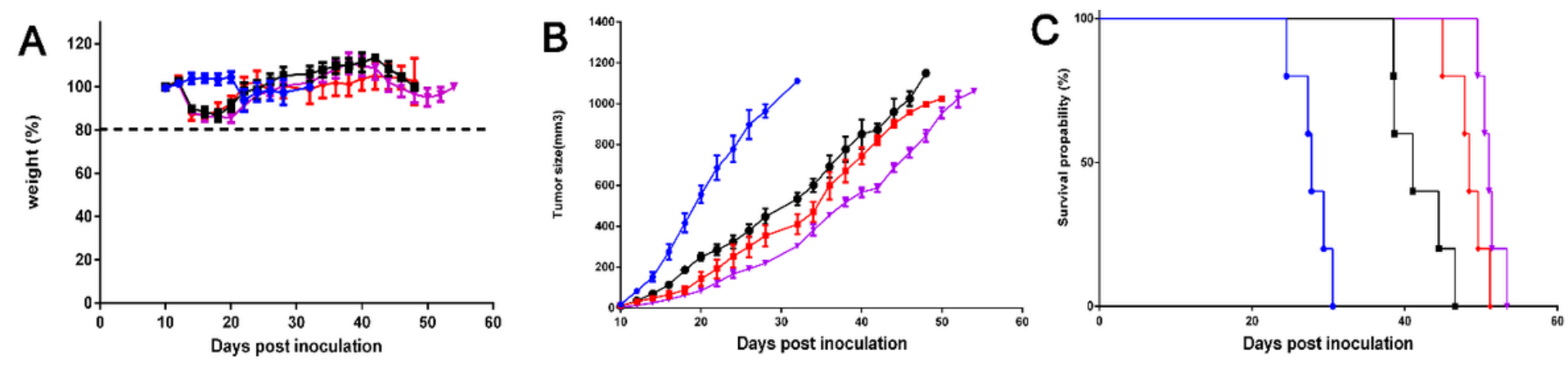

\section{- PBS * Cat-lip - Caelyx ${ }^{\circledR}$ - PEG-Azo-Cat-lip}

Figure 9 
Antitumor activities of the formulation. (A) The results of body weight over the 55 days of the study. (B) The results of the tumor volume of each group during the study. (C) The survival plot obtained by the Kaplan-Meier survival analysis. The data are presented as mean \pm SD.

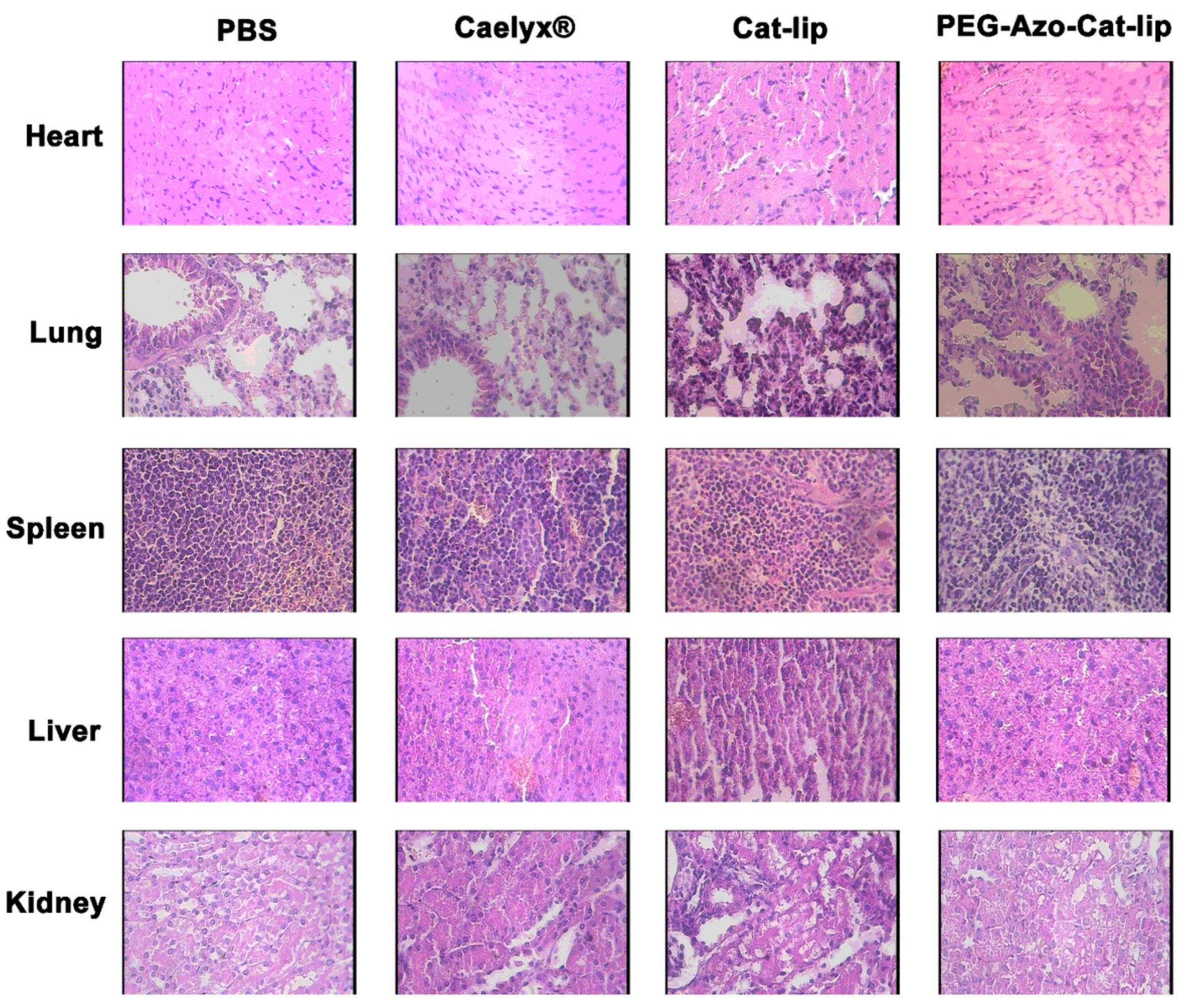

Figure 10

Histological evaluations the major organs. The biosafety of the formulations was investigated on major organs. The evaluation was performed on day 20 after i.v. injection of the formulations. The images were taken under $400 \mathrm{X}$ magnification field. 